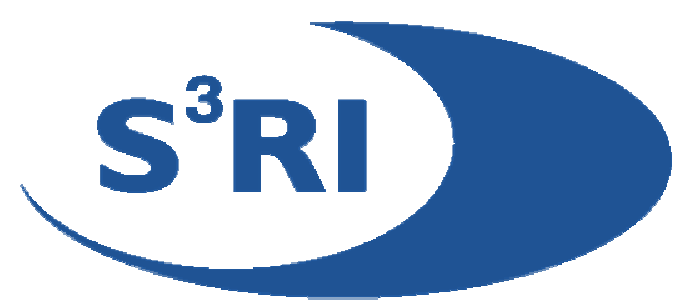

\title{
BIRTH INTERVALS AND INJECTABLE CONTRACEPTION IN SUB-SAHARAN AFRICA
}

\section{KANDALA NGIANGA-BAKWI, R. WiLliAM STONES}

\begin{abstract}
The interval between births is associated with child survival in the developing world. We investigate associations between use of depot medroxyprogesterone acetate and other reversible contraception and short birth intervals in sub-Saharan Africa. Data from successive Demographic and Health Surveys undertaken in nine African countries were analysed. Logistic regression was used to explain changes in the proportion of short birth intervals in four countries with relatively high use of reversible contraception. The overall odds ratio for the trend was 0.90 (95\%CI 0.84 to 0.95 ) and this was unaffected by adjusting for the other variables. The odds of a short birth interval were reduced by exclusive breast feeding (OR $0.67,95 \%$ CI 0.58 to 0.78 ) and increased by use of injectable contraception (OR 1.23, 95\% CI 1.11 to 1.38). The proportion of short birth intervals has changed little over the last decade in a context of very low use of the intrauterine device. Widespread adoption of injectable contraception is associated with greater odds of a short birth interval, thus not contributing favourable conditions for improved child health.
\end{abstract}

\section{Southampton Statistical Sciences Research Institute Applications \& Policy Working Paper A04/24}




\title{
Birth intervals and injectable contraception in sub-Saharan Africa
}

\author{
Kandala Ngianga-Bakwi \\ R William Stones* \\ 'Opportunities and Choices’ Programme of Reproductive Health Research \\ Southampton Statistical Sciences Research Institute \\ University of Southampton
}

*Correspondence:

Level F(815)

Princess Anne Hospital

Southampton SO16 5YA, UK

r.w.stones@soton.ac.uk

Voice +442380796033

Fax +442380786933 


\section{Introduction}

The interval between births is associated with child survival in the developing world. Short birth intervals contribute to mortality risk extending beyond the first year of life and the effect is apparent even after taking into account other potential determinants such as maternal ill health or access to health services, and allowing for the uneven quality of data available for use in multivariate analyses (1). Contraceptive use is anticipated to contribute to a reduction in the proportion of short birth intervals but evidence for this at the population level is lacking. Furthermore, while the impact on birth intervals of different reversible contraceptive methods is unknown, depot medroxyprogesterone acetate (DMPA) has gained considerable popularity in sub-Saharan Africa. We aimed to investigate associations between the proportion of short birth intervals and use of this and other reversible contraception in the region.

\section{Methods}

Data from successive Demographic and Health Surveys (2) undertaken in nine subSaharan African countries were used: Burkina Faso (1992 and 1999), Cameroon (1991 and 1998), Ghana (1993 and 1998), Kenya (1993 and 1998), Madagascar (1992 and 1997), Malawi (1992 and 2000), Niger (1992 and 1998), Tanzania (1992, 1996 and 1999), and Zambia (1992, 1996 and 2001). The percentages of women reporting a preceding birth interval of $\leq$ or $>24$ months were tabulated by country and survey date with the percentages reporting current use of any modern reversible contraception, DMPA and the intrauterine device. As the questionnaire items were very similar across surveys in the different countries it was possible to combine the data sets for further 
analysis. Pooled data from the four countries where use of reversible contraception is substantial were used to examine associations among individual women between short birth intervals and demographic and behavioural variables. To allow comparisons over time in the pooled data, a variable was created to identify data from surveys carried out during 1991-1993 and those carried out in 1999-2001. Multiple logistic regression analysis was used to calculate the crude odds ratio of having a birth interval of $\leq 24$ months during 1991-3 (reference group) and 1999-2001. We then adjusted for contraceptive choice, breastfeeding practice, mother's age, residence in urban or rural areas, education and household economic status. For the last variable we used principal components analysis to obtain an index from household ownership of certain assets (3). We experimented with interaction terms which proved non-significant.

\section{Results}

Table 1 shows that there have been small changes in the percentage of birth intervals $\leq 24$ months reported during the five to nine years between first and last surveys in the nine countries, ranging from a decrease of 6.5\% (Cameroon and Madagascar) to an increase of 6.4\% (Ghana). Over the same period use of modern methods of reversible contraception fell in Burkina Faso (-0.4\%) but rose in the other countries with Malawi showing the greatest increase of $15.3 \%$. Use of intrauterine contraception was low in the initial surveys but fell further in all countries. In the four countries with relatively high use of reversible contraception there were significant associations between short birth intervals and a range of variables (Table 2). In multiple logistic regression analysis the overall odds ratio for the trend over time was 0.90 (95\%CI 0.84 to 0.95 ) and this was unaffected 
by adjusting for the other variables. The odds of a short birth interval were reduced among those exclusively breast feeding (OR $0.67,95 \%$ CI 0.58 to 0.78 ) and increased by use of injectable contraception (OR 1.23, 95\% CI 1.11 to 1.38) (Table 3).

\section{Discussion}

Our results show that use of modern reversible contraception in the region is low, and where use has increased substantially as in Malawi, Tanzania and Zambia this has not been accompanied by any impact on the percentage of short birth intervals. Progress towards attainment of the Millennium Development Goals for child mortality is well behind target in sub-Saharan Africa. This has been ascribed to the presence of civil unrest in some countries and to the burden of the HIV epidemic (4). These risks are magnified by around one fifth of births still occurring at an interval of less than two years, and the data clearly show the very limited progress towards birth spacing in most countries over a decade. Provision of effective reversible contraception is critical in a setting where breastfeeding advice and behaviour are changing through interventions to prevent maternal to child transmission of HIV; breastfeeding duration has long been recognised as the major determinant of birth interval (5) and our findings demonstrate its continued importance. Between the two survey periods the percentage of mothers of children under three who reported no breastfeeding rose slightly from $39 \%$ to $44 \%$, perhaps reflecting changing feeding advice in the context of maternal HIV infection.

The proliferation of injectable contraception in Africa may carry some risk to women in a context of high HIV incidence: in cohort studies of commercial sex workers, use of DMPA was associated with both an increased risk of HIV infection $(6,7)$ and a 
higher viral load at the time of infection (8). However, in a cohort of women from a general population setting of whom around 15\% were DMPA users, no increase in the risk of seroconversion was seen (9) and the excess risk seen among sex workers may reflect other confounding factors. Our findings from the present analysis of sample surveys that are nationally and regionally representative show that the use of injectable contraception is associated adversely with birth interval, thus not contributing any potential child survival benefit. The pathways underlying this statistical association are not clear and require further study. It may even be that women who have experienced an unintended short birth interval are preferentially motivated to use a reliable method such as DMPA. This possibility is however not supported by examination of data from the 2000 DHS Malawi survey, where 49\% of women using DMPA reported that their last child had been "wanted then", identical to the response among those using other methods of contraception but fewer than among non-users of contraception (57\%). Whatever the nature of the association and notwithstanding its other advantages, it is clear that at the population level DMPA is not providing the anticipated increase in birth interval that would be conducive to bearing and raising healthy children.

Our study highlights the very low and declining use of intrauterine contraception in the region. This decline may have come about through the concern of providers not to recommend a method potentially associated with pelvic infection. International guidelines had restrictive medical eligibility criteria but have recently been revised with regard to advice about STI risk and HIV status (10). Research is needed to assess whether renewed emphasis on the IUD among a broad mix of contraceptive methods has potential to reduce the proportion of short birth intervals in Africa. Finally, it has been recognised 
that information provision about condom use as a "routine" method of contraception within stable unions may remove the negative implication of infidelity inseparable from condom promotion for HIV prevention: modelling of the likely impact of condom use supplanting oral contraceptives did not suggest a likely adverse impact on the numbers of unwanted, mistimed, and wanted births (11). Further work is required to obtain direct evidence of the impact of widespread condom use on the proportion of short birth intervals in Africa: such evidence would represent another positive reason for condom use within stable unions.

\section{Acknowledgement}

This study was funded by the UK Department for International Development. The funding body had no role in the design or conduct of the study or in preparation of the manuscript.

\section{Authorship contributions and confirmation}

Both authors have access to all data in the study and hold final responsibility for the decision to submit for publication. Both authors designed the study, KNB carried out the analysis and both authors wrote the manuscript. 


\section{References}

(1) Defo BK. Effects of infant feeding practices and birth spacing on infant and child survival: A reassessment from retrospective and prospective data. Journal of Biosocial Science 1997; 29:303-326.

(2) MEASURE DHS+. Demographic and Health Surveys. Providing information for informed decisions in population, health, and nutrition. www.measuredhs.com (accessed 28-5-2004).

(3) Filmer D, Pritchett LH. Estimating wealth effects without expenditure data-or tears: an application to educational enrollments in states of India. Demography 2001; 38: 115-132.

(4) The World Bank Group. Millennium Development Goals 4: Reduce child mortality. http://www.developmentgoals.org/Child_Mortality.htm (accessed 28-5-2004).

(5) Smith DP. Breastfeeding, contraception and birth intervals in developing countries. Studies in Family Planning 1985; 16:154-163.

(6) Martin HL, Nyange PM, Richardson BA, Lavreys L, Mandaliya K, Jackson DJ, Ndinya-Achola JO, Kreiss J. Hormonal contraception, sexually transmitted diseases, and risk of heterosexual transmission of human immunodeficiency virus type 1 . Journal of Infectious Diseases 1998; 178: 1053-1059.

(7) Lavreys L, Baeten JM, Martin HL, Overbaugh J, Mandaliya K, Ndinya-Achola JO, Kreiss JK. Hormonal contraception and risk of HIV-1 acquisition: results of a 10year prospective study. AIDS 2004; 18: 695-697.

(8) Lavreys L, Baeten JM, Kreiss JK, Richardson BA, Chohan BH, Hassan W, Panteleeff DD, Mandaliya K, Ndinya-Achola JO, Overbaugh J. Injectable contraceptive use and genital ulcer disease during the early phase of HIV-1 infection increase plasma virus load in women. Journal of Infectious Diseases 2004; 189: 303311.

(9) Kiddugavu M, Makumbi F, Wawer MJ, Serwadda D, Sewankambo NK, WabwireMangen F, Lutalo T, Meehan M, Xianbin, Gray RH. Hormonal contraceptive use and HIV-1 infection in a population-based cohort in Rakai, Uganda. AIDS 2003; 17: 233-240.

(10) Department of Reproductive Health and Research (RHR) WHO. Medical Eligibility Criteria for Contraceptive Use, Third Edition Summary Tables. http://www.who.int/reproductive-health/publications/MEC_3/index.htm (accessed 28-5-2004).

(11) Ali MM, Cleland J, Shah IH. Condom use within marriage: a neglected HIV intervention. Bulletin of the World Health Organisation 2004; 82: 180-186. 
Table 1: Preceding birth interval, Demographic and Health Surveys.

\begin{tabular}{|c|c|c|c|c|c|c|}
\hline \multirow[t]{2}{*}{$\overline{\text { Country }}$} & \multirow[t]{2}{*}{ Year of survey } & \multicolumn{2}{|c|}{ Birth Interval \% (No) } & \multicolumn{3}{|c|}{ Modern Reversible Contraception } \\
\hline & & $\leq 24$ months & $>24$ months & All methods \% (No) & Injectables \% & IUD \% \\
\hline \multirow[t]{2}{*}{ Burkina Faso } & 1992 & $17.6(821)$ & $82.5(3858)$ & $6.1(357)$ & 0.3 & 1.0 \\
\hline & 1999 & $19(925)$ & 81 (3946) & 5.7 (337) & 1.2 & 0.3 \\
\hline \multirow[t]{2}{*}{ Cameroon } & 1991 & $28(746)$ & 72 (1923) & $5.4(180)$ & 0.5 & 0.6 \\
\hline & 1998 & 21.5 (377) & 78.5 (1380) & $6.5(151)$ & 0.9 & 0.2 \\
\hline \multirow[t]{2}{*}{ Ghana } & 1993 & $14.9(260)$ & 85.1 (1489) & $7.9(174)$ & 1.1 & 0.4 \\
\hline & 1998 & $21.3(705)$ & 78.7 (2605) & $11.4(370)$ & 3.6 & 0.3 \\
\hline \multirow[t]{2}{*}{ Kenya } & 1993 & 30.6 (1494) & 69.4 (3383) & 20.6 (1257) & 7.6 & 2.2 \\
\hline & 1998 & $26.7(707)$ & 73.3 (1939) & $21.9(773)$ & 10.5 & 1.0 \\
\hline \multirow[t]{2}{*}{ Madagascar } & 1992 & 35.1 (1461) & 64.9 (2706) & $4.3(225)$ & 1.7 & 0.4 \\
\hline & 1997 & $28.6(812)$ & 71.4 (2026) & 7.5 (275) & 4.4 & 0.2 \\
\hline \multirow[t]{2}{*}{ Malawi } & 1992 & $23.9(871)$ & 76.1 (2781) & 7.9 (357) & 1.6 & 0.3 \\
\hline & 2000 & 20.7 (1861) & $79.3(7141)$ & $23.2(2772)$ & 18.2 & 0.1 \\
\hline \multirow[t]{2}{*}{ Niger } & 1992 & 29.8 (1743) & 70.2 (4100) & $6.4(443)$ & 1.5 & 0.5 \\
\hline & 1998 & $23.6(920)$ & 76.4 (2981) & $7.3(349)$ & 2.2 & 0.2 \\
\hline \multirow[t]{3}{*}{ Tanzania } & 1992 & 21.3 (1349) & 78.7 (4971) & $4.9(402)$ & 5.9 & 0.3 \\
\hline & 1996 & 20.1 (1066) & 79.9 (4250) & 14.9 (1009) & 7.4 & 0.4 \\
\hline & 1999 & $21.5(538)$ & 78.5 (1961) & $16.8(541)$ & 0.2 & 0.5 \\
\hline \multirow[t]{3}{*}{ Zambia } & 1992 & 22.8 (1121) & 77.2 (3786) & $7.6(480)$ & 0.2 & 0.3 \\
\hline & 1996 & 22.9 (1297) & 77.1 (4357) & $12.5(906)$ & 0.9 & 0.1 \\
\hline & 2001 & $19.9(1065)$ & 80.1 (4282) & $18.1(1241)$ & 4.2 & 0.03 \\
\hline
\end{tabular}


Table 2: Short birth interval (<24 months) by selected characteristics, Kenya, Malawi, Tanzania and Zambia. Bivariate analyses of unadjusted data.

\begin{tabular}{l|lll}
\hline Variable & $\%$ & No & $P>|Z|$ \\
\hline Year (1999-2001) & 16.3 & 25549 & 0.000 \\
Year (1991-1993) & 19.3 & 25047 reference category & \\
Urban & 15.4 & 10939 & 0.000 \\
Rural & 18.4 & 39657 reference category & \\
No education & 18.9 & 13063 & 0.000 \\
Primary education & 17.7 & 31124 & 0.000 \\
Secondary Education and higher & 16.0 & 6407 reference category & \\
Exclusive breastfeeding & 12.6 & 1753 & 0.000 \\
Mixed feeding & 17.0 & 24573 & 0.000 \\
No breastfeeding & 18.0 & 18711 reference category & \\
Low income households & 18.4 & 17749 & 0.000 \\
Middle income households & 18.8 & 20768 & 0.000 \\
High income households & 15.2 & 12079 reference category & \\
Young mothers (<21 years old) & 12.8 & 13397 & 0.000 \\
Old mothers (>35 years old) & 15.2 & 5462 & 0.000 \\
Mothers (> 21 and <35 years old) & 20.3 & 31737 reference category & \\
No contraception use & 17.3 & 37964 & 0.000 \\
Using injections & 21.7 & 3612 & 0.000 \\
Using other methods & 18.4 & 9020 reference category & \\
\hline Total & 17.8 & 50596 & \\
\hline
\end{tabular}


Table 3: Adjusted odds ratios for short birth interval (<24 months), Kenya, Malawi, Tanzania and Zambia.

\begin{tabular}{l|llcc}
\hline Variable & Odds ratio & Std. Err & $95 \%$ CI & $P>|Z|$ \\
\hline Year (1999-2001) & 0.90 & 0.02 & $0.84,0.95$ & 0.000 \\
Year (1991-1993) & 1 & \multicolumn{4}{c}{ reference category } \\
Urban & 0.85 & \multicolumn{4}{l}{0.03} & $0.79,0.90$ & 0.000 \\
Rural & 1 & \multicolumn{4}{c}{ reference category } \\
No education & 1.16 & 0.05 & $1.06,1.26$ & 0.001 \\
Primary education & 1.11 & 0.04 & $1.03,1.20$ & 0.009 \\
Secondary Education and higher & 1 & reference category & \\
Exclusive breastfeeding & 0.67 & 0.05 & $0.58,0.78$ & 0.000 \\
Mixed feeding & 0.86 & 0.02 & $0.82,0.90$ & 0.000 \\
No breastfeeding & 1 & reference category & \\
Low income households & 1.18 & 0.04 & $1.10,1.26$ & 0.000 \\
Middle income households & 1.25 & 0.04 & $1.17,1.34$ & 0.000 \\
High income households & 1 & reference category & \\
Young mothers (<21 years old) & 0.58 & 0.02 & $0.55,0.62$ & 0.000 \\
Old mothers (>35 years old) & 0.67 & 0.03 & $0.62,0.72$ & 0.000 \\
Mothers (> 21 and <35 years old) & 1 & reference category & \\
No contraception use & 0.88 & 0.03 & $0.82,0.93$ & 0.000 \\
Using injections & 1.23 & 0.07 & $1.10,1.38$ & 0.000 \\
Using other methods & 1 & reference category & \\
\hline Interaction (injection*low income) & 1.13 & 0.10 & $0.18,1.35$ & 0.316 \\
\hline
\end{tabular}

